\title{
On the Lambert-W function for constrained resource allocation in cooperative networks
}

\author{
Félix Brah ${ }^{1 *}$, Abdellatif Zaidi ${ }^{2}$, Jérôme Louveaux ${ }^{1}$ and Luc Vandendorpe ${ }^{1}$
}

\begin{abstract}
In cooperative networks, a variety of resource allocation problems can be formulated as constrained optimization with system-wide objective, e.g., maximizing the total system throughput, capacity or ergodic capacity, subject to constraints from individual users, e.g., minimum data rate, transmit power limit, and from the system, e.g., power budget, total number of subcarriers, availability of the channel state information (CSI). Most constrained resource allocation schemes for cooperative networks require rigorous optimization processes using numerical methods since closed-form solutions are rarely found. In this article, we show that the Lambert-W function can be efficiently used to obtain closed-form solutions for some constrained resource allocation problems. Simulation results are provided to compare the performance of the proposed schemes with other resource allocation schemes.
\end{abstract}

Keywords: Resource allocation, Lambert-W function, cooperative networks, QoS

\section{Introduction}

Cooperative transmissions have attracted much attention over the last few years. It has been demonstrated that the benefits of multi-antenna transmission can be achieved by cooperative transmission without requiring multiple antennas at individual nodes (for example see [1-3]). Cooperation is particularly relevant when the size of mobile devices limits the number of antennas that can be deployed.

Wireless Mesh networks (WMN) and relay networks are among the main networks that use cooperative communication. The main distinguished characteristic of mesh and relay networks is possibility of multi-hop communication. In Mesh networks, traffic can be routed through other mobile stations (MSs) and can also take place through direct links. Nodes are composed of mesh routers and mesh clients and thus routing process is controlled not only by base station (BS) but also by mobile station MS [4]. Each node can forward packets on behalf of other nodes that may not be within direct wireless transmission range of their destination. In case of relay networks, the network infrastructure consists of relay stations (RSs) that are mostly installed, owned, and

\footnotetext{
* Correspondence: felix.brah@uclouvain.be

${ }^{1}$ ICTEAM Institute, Université Catholique de Louvain, Place du Levant 2, 1348 Louvain-la-Neuve, Belgium

Full list of author information is available at the end of the article
}

controlled by service provider. A RS is not connected directly to wire infrastructure and has the minimum functionality necessary to support multi-hop communication. The important aspect is that traffic always leads from or to BS. The realization of the performance improvement promised by cooperation in wireless mesh and relay networks depends heavily on resource allocation (among other things).

Recently, resource allocation for OFDMA WMN with perfect CSI has been an active research topic. In [5], a fair subcarrier and power allocation scheme to maximize the Nash bargaining fairness has been proposed. Instead of solving a centralized global optimization problem, the authors proposed a distributed hierarchical approach where the mesh router allocates groups of subcarriers to the mesh clients, and each mesh client allocates transmit power among its subcarriers to each of its outgoing links. In [6], an efficient intra-cluster packet-level resource allocation approach taking into account power allocation, subcarrier assignment, packet scheduling, and QoS support has been studied. The authors employ the utility maximization framework to find the joint powerfrequency-time resource allocation that maximizes the sum rate of a WMN while satisfying users minimum rate requirements. The benefits of optimal resource allocation in cooperative relay networks with perfect CSI

\section{SpringerOpen ${ }^{\circ}$}

(C) 2011 Brah et al; licensee Springer. This is an Open Access article distributed under the terms of the Creative Commons Attribution License (http://creativecommons.org/licenses/by/2.0), which permits unrestricted use, distribution, and reproduction in any medium, provided the original work is properly cited. 
has also been investigated by several authors (see, e.g., $[7,8]$, and references therein).

However, when the channel variations are fast, the transmitter may not be able to adapt to the instantaneous channel realization. Hence, CSI-aware resource allocation is not suitable for environments with high mobility. When the channel state can be accurately tracked at the receiver side, the statistical channel model at the transmitter can be based on channel distribution information feedback from the receiver. We refer to knowledge of the channel distribution at the transmitter as CDIT. In [9], CDIT-based constrained resource allocation problem for non-cooperative OFDMA-based networks is studied. The authors derive an optimal power allocation algorithm in closed-form. In [10], a dynamic resource allocation algorithm aiming to maximize the delay-limited capacity of a cooperative communication with statistical channel information is developed. In [11], a power allocation problem for ergodic capacity maximization in relay networks under high SNR regime is solved using numerical methods.

In this article, we present a new result on how the Lambert-W function can be used to efficiently find closed-form solution of constrained resource allocation problems for cooperative networks.

There are two significant benefits from using the Lambert-W function in the context of resource allocation for cooperative networks. Most resource allocation schemes for cooperative networks require rigorous optimization processes using numerical methods since closed-form solutions are rarely found. Using the Lambert-W function, resource allocations can not only be expressed in closed-form but they can also quickly be determined without resorting to complex algorithms since a number of popular mathematical softwares, including Maple and Matlab, contain the Lambert-W function as an optimization component.

The Lambert-W function has several uses in physical and engineering applications [12-15]. In [14], the Lambert-W function is used for the purpose of diode parameters determination in diode $I-U$ curve fitting. Recent work in [15] shows that the Lambert-W function also finds utilization in Astronomy to calculate the position of an orbiting body in a central gravity field.

The remainder of this article is organized as follows. In Sect. 2, we provide a concise introduction to the Lambert-W function. In Sect. 3, we show how the Lambert-W function is applied to a subcarrier allocation problem in WMNs. The use of the Lambert-W function to a power allocation problem in relay networks with statistical channel information is discussed in Sect. 4. In Sect. 5, we show the performance of the proposed resource allocation methods by simulation. Finally, conclusions are drawn in Sect. 6.

\section{The Lambert-W Function}

The Lambert function $W(x)$ is defined to be the multivalued inverse of the function $f(x)=x e^{x}$ [12]. That is, Lambert $\mathrm{W}(\mathrm{x})$ can be any function solution of the transcendental equation

$$
W(x) e^{W(x)}=x .
$$

Actually, for some values of $x$, Equation 1 has more than one root, in which case the different solutions are called branches of $W$. Since the values of interest in our work are real, we will concentrate on real-value branches of $W$. If $x$ is real, then for $-1 / e \leq x<0$, there are two possible real values of $W(x)$ (see Figure 1). The branch satisfying $W(x) \leq-1$ is denoted by $W_{0}(x)$. The branch satisfying $W(x) \leq-1$ is denoted by $W(x)$ and it is referred to as the principal branch of the Lambert-W function.

The $n$th derivative of the Lambert-W function is given by [12].

$$
\frac{\mathrm{d}^{n} W\left(e^{x}\right)}{\mathrm{d} x^{n}}=\frac{q_{n}\left(W\left(e^{x}\right)\right)}{\left(1+W\left(e^{x}\right)\right)^{2 n-1}} \quad \text { for } n \geq 1,
$$

in which the polynomials $q_{n}$, given by

$$
q_{n}(w)=\sum_{i=0}^{n-1}\left\langle\left\langle\begin{array}{c}
n-1 \\
i
\end{array}\right)\right\rangle(-1)^{i} w^{i+1},
$$

contain coefficients expressed in terms of secondorder Eulerian numbers and $q_{1}(w)=w$.

In (3), the second-order Eulerian number $\left\langle\left(\begin{array}{c}n \\ m\end{array}\right\rangle\right)$ corresponds to the number of permutations of the multiset $\{1,1,2,2, \ldots, n, n\}$ with $m$ ascents which have the property that for each $i$, all the numbers appearing between the two occurrences of $i$ in the permutation are greater than $i[16]$.

The application of the Lambert-W function to obtain a closed-form solution for resource allocation problems in wireless mesh and relay networks constitutes the principal contribution of this article.

\section{The Lambert-W function for subcarrier allocation in wireless mesh network}

\subsection{Problem formulation}

We consider a single cluster OFDMA WMN that consists of one mesh router (MR) and $K$ mesh clients (MC) as illustrated in Figure 2. The MR serves as a gateway for the MCs to the external network (e.g., Internet). The MCs can communicate with the MR and with each other through multi-hop routes via other MCs. We label the MR as node 0 and the MC nodes as $k=1, \ldots, K$. A link $(k, j)$ exists between node $k$ and node $j$ when they are within transmission range of each other, i.e., they are neighboring nodes. 


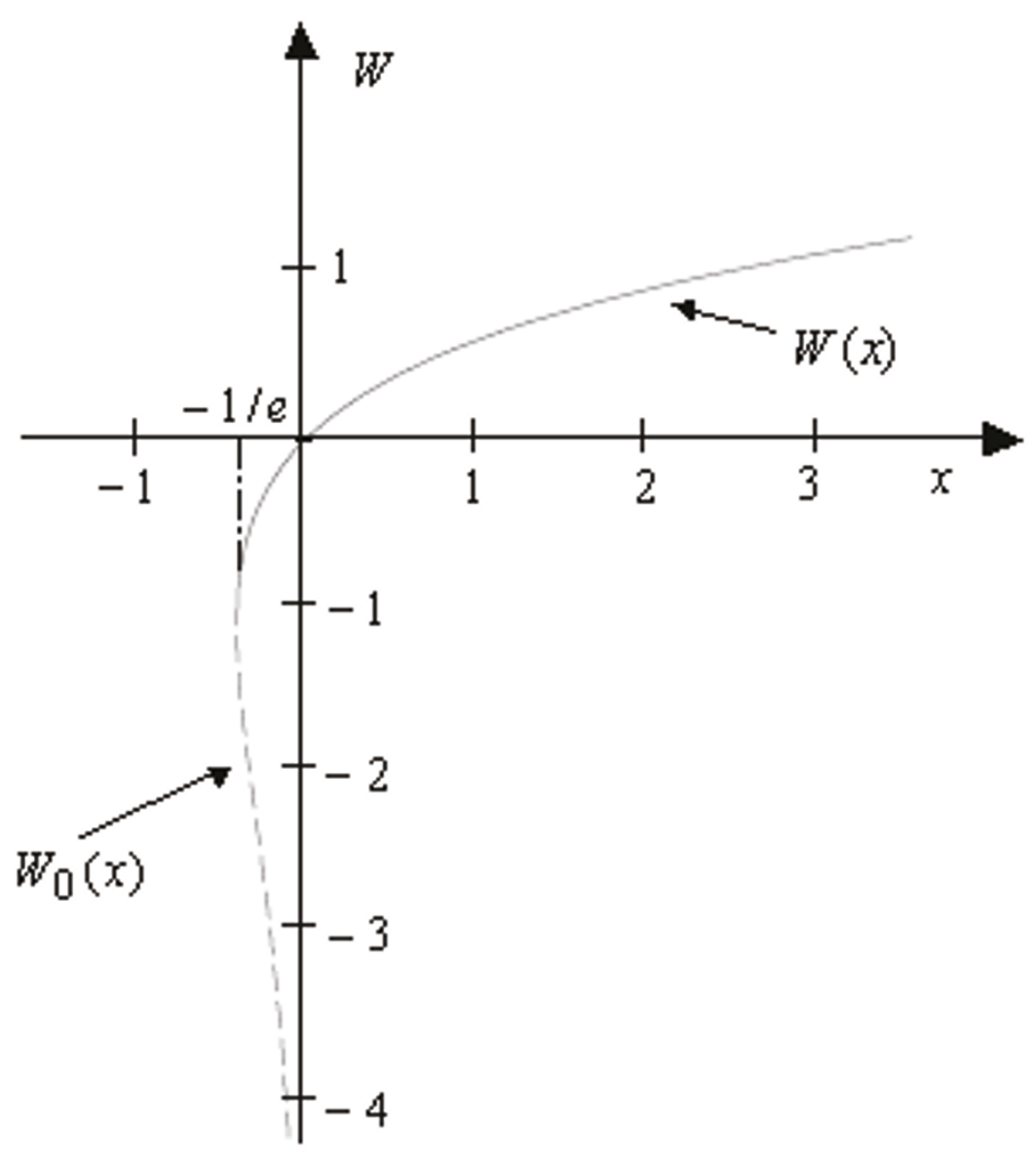

Figure 1 The two real branches of the Lambert-W function.

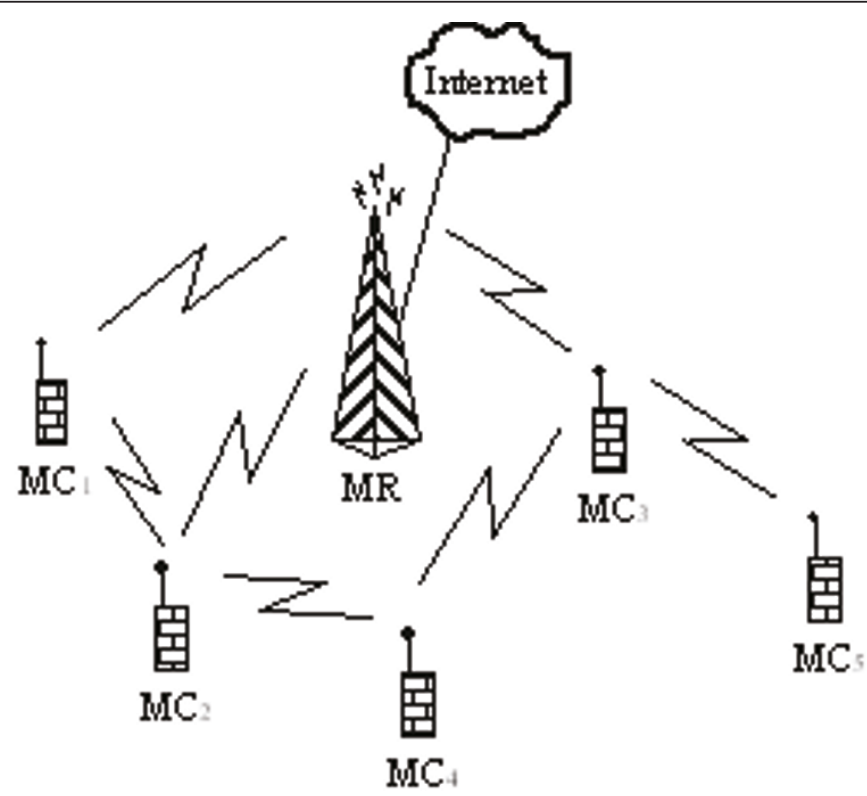


There are a total of $N$ subcarriers in the system. Each subcarrier has a bandwidth $B$. The channel gain of subcarrier $n$ on link $(k, j)$, which connects MC $k$ to MC $j$, is denoted by $G_{k j}^{n}$ and the transmit power of MC $k$ on subcarrier $n$ is denoted by $p_{k}^{n}$. MC $k$ has a transmit power limit of $\bar{p}_{k}$ and a minimal rate requirement of $R_{k}$. Let $n_{k}$ be the number of subcarriers to allocate to MC $k$, using only information available at the MR, i.e., the average channel gain of all outgoing links at MC $k, \bar{G}_{k}$. Based on $\bar{G}_{k}$ and uniform power allocation assumption over all the $n_{k}$ subcarriers $\left(p_{k}^{n}=\bar{p}_{k} / n_{k}, \forall k\right)$, the MR determines an approximated rate for MC $k$ as

$$
r_{k}\left(n_{k}\right)=n_{k} B \log _{2}\left(1+\frac{\bar{G}_{k}}{\Gamma \sigma_{\mathrm{n}}^{2}} \frac{\bar{p}_{k}}{n_{k}}\right),
$$

where $\sigma_{\mathrm{n}}^{2}$ is the thermal noise power, and $\Gamma$ is the SNR gap related to the required bit-error-rate (BER). The main reason that the MR determines an approximated rate instead of the exact rate is that the MR knows only the average channel gain $\bar{G}_{k}$, but not the complete channel gain $G_{k j}^{n}$. In general, exact and complete information needed to determine the exact rate is rarely available at the MR. For practical SNR values $(S N R>5 \mathrm{~dB})$, the gap between the exact rate and its approximate (4) is very small and (4) can be viewed as the rate realized at MC $k$.

There are various constraints associated with resource allocation in OFDMA-based WMNs. At each node $k$, the sum of the transmit power on the allocated subcarriers is bounded by a maximum power level $\bar{p}_{k}$. We assume that each subcarrier can only be allocated to one transmission link in a cluster. Different traffic types require different packet transmission rates. For example, voice packets require a constant rate; video traffic has minimum, mean, and maximum rate requirements; while data traffic is usually treated as background traffic whose source rate is dynamic. In our problem formulation, we only take the minimum rate requirement of these three traffic types, if any, into account.

The resources to allocated are defined as a set of subcarriers, and the total transmit power available at each node. We consider a distributed hierarchical resource allocation, where the MR only performs a rough resource allocation with limited information (the average channel gain of all outgoing links at MC or the statistical channel information) and the $\mathrm{MCs}$ perform more refined resource allocation with full information that is available locally. In this section, we focus on subcarrier allocation at MR and we assume that each MC $k$ distributes its transmit power limit $\bar{p}_{k}$ equally over all its allocated subcarriers. After subcarrier allocation, the optimal power allocation is performed at each MC $k$. The optimal power allocation is not developed in this article. Mathematically, the subcarrier allocation problem at MR can be formulated as

$$
\max _{n_{k}} \sum_{k=1}^{K} n_{k} B \log _{2}\left(1+\frac{\alpha_{k}}{n_{k}}\right)
$$

subject to:

$$
\begin{aligned}
& n_{k} B \log _{2}\left(1+\frac{\alpha_{k}}{n_{k}}\right) \geq R_{k} \\
& \sum_{k=1}^{K} n_{k} \leq N
\end{aligned}
$$

where $\alpha_{k}=\frac{\bar{G}_{k} \bar{p}_{k}}{\Gamma \sigma_{n}^{2}}$.

\subsection{Solution method}

We propose a solution method based on the Lagrange dual approach and the Lambert-W function. First, we express the Lagrangian of the primal problem (5) as

$$
\begin{aligned}
L\left(n_{k}, \lambda_{k}, \mu\right)= & \sum_{k=1}^{K} n_{k} B \log _{2}\left(1+\frac{\alpha_{k}}{n_{k}}\right) \\
& +\sum_{k=1}^{K} \lambda_{k}\left(n_{k} B \log _{2}\left(1+\frac{\alpha_{k}}{n_{k}}\right)-R_{k}\right)-\mu\left(\sum_{k=1}^{K} n_{k}-N\right)
\end{aligned}
$$

where $\lambda_{k}$ and $\mu$ are the Lagrangian multipliers associated with the minimum rate constraint of MC $k$ and the total subcarrier constraint.

By KKT first optimality condition [17], we take the derivative of (6) with respect to $n_{k}$ for fixed $\left(\lambda_{k}, \mu\right)$ and set the derivative to zero to obtain

$$
\ln \left(1+\frac{\alpha_{k}}{n_{k}}\right)-\frac{\frac{\alpha_{k}}{n_{k}}}{1+\frac{\alpha_{k}}{n_{k}}}-\frac{\mu}{\frac{B}{\ln 2}\left(1+\lambda_{k}\right)}=0 .
$$

By solving Equation 7 for $n_{k}$, for given $\left(\lambda_{k}, \mu\right)$, the optimal value of subcarriers to be allocated to MC $k$ is given by (see Appendix A)

$$
n_{k}^{*}=\frac{-\alpha_{k} W\left(-\exp \left(-1-\frac{\mu}{\frac{B}{\ln 2}\left(1+\lambda_{k}\right)}\right)\right)}{1+W\left(-\exp \left(-1-\frac{\mu}{\frac{B}{\ln 2}\left(1+\lambda_{k}\right)}\right)\right)} .
$$

The optimal values of $\mu$ and $\lambda_{k}$ still need to be found. They correspond to the ones that satisfy the total subcarrier constraint with equality and the individual rate constraints. We substitute $n_{k}$ in Equation 6 by $n_{k}^{*}$ to form the dual problem 


$$
\min _{\lambda_{k}, \mu} L\left(n_{k}^{*}, \lambda_{k}, \mu\right) .
$$

The optimal $\lambda_{k}^{*}$, for fixed $\mu$, are found using KKT conditions. To derive $L\left(n_{k}^{*}, \lambda_{k}, \mu\right)$ over $\lambda_{k}$, we make use of the formula of the $n$th derivative of the Lambert-W function given by (2). Applying (2) for $n=1$, we obtain that the optimal $\lambda_{k}^{*}$ has to satisfy (see Appendix B)

$$
f_{m}\left(\lambda_{k}^{*}\right)-\frac{R_{k}}{\alpha_{k}}=0
$$

where

$$
\begin{aligned}
f_{m}\left(\lambda_{k}^{*}\right)= & \frac{w_{k}^{*}}{1+w_{k}^{*}} \cdot\left[\left(\frac{B}{\ln 2}+\frac{\mu}{\left(1+\lambda_{k}^{*}\right)\left(1+w_{k}^{*}\right)^{2}}\right) \ln \left(-w_{k}^{*}\right)\right. \\
& \left.+\frac{\mu}{\left(1+\lambda_{k}^{*}\right)\left(1+w_{k}^{*}\right)}+\frac{\mu^{2}}{\left(1+\lambda_{k}^{*}\right)^{2}\left(1+w_{k}^{*}\right)^{2}}\right]
\end{aligned}
$$$$
\text { with } w_{k}^{*}=W\left(-\exp \left(-1-\frac{\mu}{\frac{B}{\ln 2}\left(1+\lambda_{k}^{*}\right)}\right)\right) \text {. }
$$

It can be shown that $f_{m}$ is a strictly increasing function of $\lambda_{k}^{*}$ and $f_{m}\left(\lambda_{k}^{*}\right)>0$ for all $\lambda_{k}^{*} \geq 0$. Thus, the inverse function, $f_{m}^{-1}$, of $f_{m}$, exists. The optimal $\lambda_{k}^{*}$ can then be deduced as

$$
\lambda_{k}^{*}=f_{m}^{-1}\left(\frac{R_{k}}{\alpha_{k}}\right) .
$$

Now we turn to find the optimal $\mu^{*}$. Substituting $\lambda_{k}$ in (8) by the optimal value obtained in (11) and using the constraint $\sum_{k=1}^{K} n_{k}=N$, we obtain

$$
\sum_{k=1}^{K} \frac{-\alpha_{k} W\left(-\exp \left(-1-\frac{\mu}{\frac{B}{\ln 2}\left(1+\lambda_{k}^{*}\right)}\right)\right)}{1+W\left(-\exp \left(-1-\frac{\mu}{\frac{B}{\ln 2}\left(1+\lambda_{k}^{*}\right)}\right)\right)}=N .
$$

Let

$$
g_{m}(\mu)=\sum_{k=1}^{K} \frac{-\alpha_{k} W\left(-\exp \left(-1-\frac{\mu}{\frac{B}{\ln 2}\left(1+\lambda_{k}^{*}\right)}\right)\right)}{1+W\left(-\exp \left(-1-\frac{\mu}{\frac{B}{\ln 2}\left(1+\lambda_{k}^{*}\right)}\right)\right)} .
$$

Proposition 1 An inverse function for $g_{m}, g_{m}^{-1}$, exists (see Appendix C. for proof).

Thus

$$
\mu^{*}=g_{m}^{-1}(N)
$$

\subsection{Extension to Mesh router with statistical channe information}

In some fading environments, there may not be a feedback link sufficiently fast to convey the full CSI to the MR. The MR may know only the channel distribution information (CDI) and may use the CDI to allocate resource. Following the approach in [9], we can formulate an ergodic rate maximization problem at the mesh router with only CDI as

$$
\max _{n_{k}} E_{\alpha}\left\{\sum_{k=1}^{K} n_{k} B \log _{2}\left(1+\frac{\alpha_{k}}{n_{k}}\right)\right\}
$$

subject to:

$$
\begin{aligned}
& E_{\alpha_{k}}\left\{n_{k} B \log _{2}\left(1+\frac{\alpha_{k}}{n_{k}}\right)\right\} \geq R_{k} \\
& \sum_{k=1}^{K} n_{k} \leq N
\end{aligned}
$$

where $\alpha=\left[\alpha_{1}, \alpha_{2}, \ldots, \alpha_{k}, \ldots, \alpha_{K}\right]$, and $E_{\alpha}\{$.$\} represents$ the statistical expectation with respect to $\alpha$.

Using the solution method proposed in 3.2 , the optimal subcarrier allocation solution of (15) can be obtained by solving the following equation for $\tilde{n}_{k}$

$$
E_{\alpha_{k}}\left\{\ln \left(1+\frac{\alpha_{k}}{\tilde{n}_{k}}\right)-\frac{\frac{\alpha_{k}}{\tilde{n}_{k}}}{1+\frac{\alpha_{k}}{\tilde{n}_{k}}}-\frac{\mu}{\frac{B}{\ln 2}\left(1+\lambda_{k}\right)}\right\}=0 .
$$

To express the left hand side of (16), we need to find the probability density function (pdf) of the random variable

$$
\tilde{f}_{m}\left(\alpha_{k}\right)=\ln \left(1+\frac{\alpha_{k}}{\tilde{n}_{k}}\right)-\frac{\frac{\alpha_{k}}{\tilde{n}_{k}}}{1+\frac{\alpha_{k}}{\tilde{n}_{k}}}-\frac{\mu}{\frac{B}{\ln 2}\left(1+\lambda_{k}\right)} .
$$

It can be observed that $\tilde{f}_{m}$ is monotonically nondecreasing and non-negative with respect to $\alpha_{k}$. Thus, there exists a unique inverse function, $\tilde{f}_{m}^{-1}$, of $\tilde{f}_{m}$.

Let $\tilde{F}_{\alpha_{k}}\left(\alpha_{k}\right)$ and $\tilde{T}_{\alpha_{k}}\left(\alpha_{k}\right)$ denote the cumulative distribution function (cdf) and the pdf of $\alpha_{k}$. We assume that $\tilde{F}_{\alpha_{k}}\left(\alpha_{k}\right)$ and $\tilde{T}_{\alpha_{k}}\left(\alpha_{k}\right)$ are known at the MR.

First, using the same derivation as in Appendix A where the right hand side of equation (A.1) is $\tilde{f}_{m}$ instead of 0 , we can express the inverse function of $\tilde{f}_{m}$ as

$$
\tilde{\alpha}_{k}\left(\tilde{f}_{m}\right)=\frac{-\tilde{n}_{k}^{*}\left(1+W\left(-\exp \left(-1-\frac{\mu+\tilde{f}_{m}}{\frac{B}{\ln 2}\left(1+\lambda_{k}\right)}\right)\right)\right.}{W\left(-\exp \left(-1-\frac{\mu+\tilde{f}_{m}}{\frac{B}{\ln 2}\left(1+\lambda_{k}\right)}\right)\right)} .
$$


Using expression (18) for the root, we derive the cdf of $\tilde{f}_{m}$ as

$$
\tilde{F}_{\tilde{f}_{m}}\left(\tilde{f}_{m}\right)=F_{\alpha_{k}}\left(\tilde{\alpha}_{k}\left(\tilde{f}_{m}\right)\right) \text {. }
$$

The pdf of $\tilde{f}_{m}$ is then given as the derivative of (19) with respect to $\tilde{f}_{m}$ as

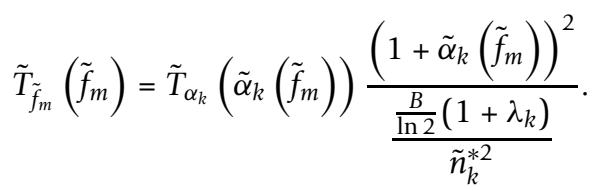

Finally, using (20), the optimal subcarrier assignment $\tilde{n}_{k}^{*}$ is obtained by solving the following equation for $\tilde{n}_{k}^{*}$

$$
\int_{0}^{\infty} \tilde{f}_{m} \tilde{T}_{\tilde{f}_{m}}\left(\tilde{f}_{m}\right) d \tilde{f}_{m}=0 .
$$

For given multipliers $\lambda_{k}$ and $\mu$, Equation 21 can be solved numerically.

The optimal values of $\lambda_{k},(k \in[1, K])$ and $\mu$ still need to be found. They correspond to the ones that satisfy the individual rate constraints and the total subcarriers constraint (with equality). If some of the individual rate constraints are exceeded, the corresponding $\lambda_{k}$ is equal to zero. Unlike in the instantaneous allocation where we have derive closed forms for $\lambda_{k}$ and $\mu$, here it is not easy to obtain a close form. We use an iterative search algorithm to find the optimal set of $\lambda_{k}$ and $\mu$.

\section{The Lambert-W function for CDIT-based power allocation in relay networks}

In this section, we show how the Lambert-W Function can be used for constrained resource allocation in relay networks.

\subsection{Problem formulation}

Consider the relay network operating in receiver cooperation mode as illustrated in Figure 3. The transmitter at the source node sends a signal $x$. Let $x_{1}$ and $y_{1}$ denote the transmitted and received signals at the relay node, respectively. We assume that the relay node operates in the full duplex mode, i.e., the relay can receive and transmit simultaneously on the same frequency channel [7]. Thus, the received signals at the relay node and the destination node are given by

$$
\begin{aligned}
& y_{1}=h_{2} x+z_{1} \\
& y=h_{1} x+\sqrt{g} h_{3} x_{1}+z,
\end{aligned}
$$

where $z_{1}$ and $z$ are independent identically distributed (i.i.d) zero mean circularly symmetric complex Gaussian (ZMCSCG) additive noise with unit variance.

The capacity cut set bound of the relay network of Figure 3 operating in a full duplex mode with perfect CSI can be expressed as [11]

$$
\begin{aligned}
C_{\text {inst }}=\max _{0 \leq \rho, \beta \leq 1} \min \left\{\log _{2}\left(1+\beta P\left(1-\rho^{2}\right)\left(\gamma_{1}+\gamma_{2}\right)\right),\right. \\
\left.\log _{2}\left(1+\beta P \gamma_{1}+((1-\beta) g+2 \rho \sqrt{\beta(1-\beta) g}) P \gamma_{3}\right)\right\},
\end{aligned}
$$

where $\rho$ represents the correlation between the transmit signals of the transmitter and the relay, and $\gamma_{i}=\left|h_{i}\right|$ 2 .

We assume Rayleigh fading where each channel gain $h_{i}, i=1,2,3$, is i.i.d. and normalized to have unit variance; hence, the corresponding channel power gain is $i$. i.d. exponential with unit mean. The average channel power gain between the relay and the receiver is $g$. We assume that $g$ characterizes only path-loss attenuation, hence $g=1 / d^{\alpha}$, where $d$ is the distance between the relay node and the receiver node and $\alpha$ is the path-loss power attenuation exponent. As in receiver cooperation mode the relay is assumed to be close to the receiver, the scenario of interest is when $d$ is small.

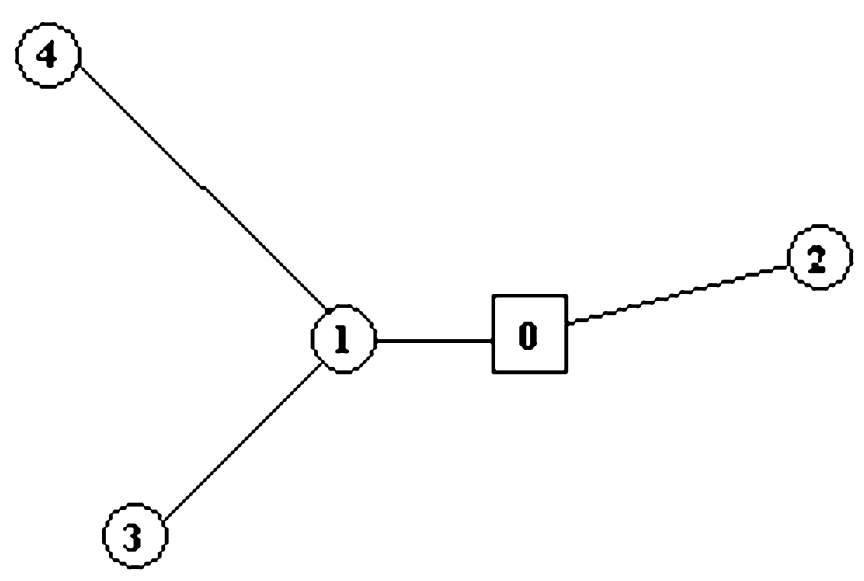

Figure 3 Illustration of a relay network operating in receiver cooperation mode. 
We consider a fast fading environment, where the receiver has CSI to perform coherent detection, but there is no fast feedback link to convey the CSI to the transmitter. Hence, the transmitter only has CDI, but no knowledge of the instantaneous channel realizations. Ergodic capacity is used to characterize the transmission rate of the channel.

We assume the channel has unit bandwidth. We further assume an average network power constraint on the system:

$$
E\left[|x|^{2}+\left|x_{1}\right|^{2}\right] \leq P,
$$

where the expectation is taken over repeated channel uses.

The network power constraint model is applicable when the node configuration in the network is not fixed [11]. Note that, when the node configuration is fixed, the individual power constraint model reflects the practical scenarios more than the network model. However, the power allocation problem is, in general, more tractable under network power constraint.

The total power $P$ is optimally allocated between the transmitter and the relay, i.e.,

$$
E\left[|x|^{2}\right] \leq \beta P, \quad E\left[\left|x_{1}\right|^{2}\right] \leq(1-\beta) P,
$$

where $\beta \in[0,1]$ is parameter to be optimized based on $\mathrm{CDI}$ and node geometry $g$.

It has been shown in [7] that the capacity upper bound in the asynchronous channel model, i.e., the channel model where the nodes do not have complete CSI, can be found by setting the correlation $\rho$ to zero. Since the CDI channel model falls into this case, the ergodic capacity upper bound can be found by taking the expectation of (23) over the channel distribution and setting $\rho=0$. Making use of the high SNR $(P \gg 1)$ approximation $\log (1+x P) \approx \log (x P)$, the ergodic capacity upper bound is then given by

$$
\begin{aligned}
& C_{\text {erg }}=\max _{0 \leq \beta \leq 1} \min \left\{E\left[\log _{2}\left(\beta P\left(\gamma_{1}+\gamma_{2}\right)\right)\right],\right. \\
&\left.E\left[\log _{2}\left(\beta P \gamma_{1}+(1-\beta) P g \gamma_{3}\right)\right]\right\} .
\end{aligned}
$$

The problem is to find the optimal power allocation, i. e., the optimal value of $\beta$, that gives the capacity upper bound $C_{\text {erg }}(\beta)$ of (26). Mathematically the power allocation problem can be formulated as

$$
\begin{aligned}
\max _{0 \leq \beta \leq 1} \min \left\{E\left[\log _{2}\left(\beta P\left(\gamma_{1}+\gamma_{2}\right)\right)\right],\right. \\
\left.E\left[\log _{2}\left(\beta P \gamma_{1}+(1-\beta) P g \gamma_{3}\right)\right]\right\} .
\end{aligned}
$$

Problem (27) has been addressed in [11] and a numerical solution has been proposed, but no closed-form expression has been provided. This contrasts with what will be done here.

\subsection{Solution method}

To find the optimal power allocation in closed-form, we propose an approach that uses the Lambert-W function.

First, we need to evaluate the expected value of the capacity expression over the channel fading distribution. For this end, we make use of the following formula for i.i. d. exponential random variables $X_{1}, X_{2}$ with unit mean:

$$
\begin{aligned}
& E\left[\log \left(a_{1} X_{1}+a_{2} X_{2}\right)\right] \\
& \quad= \begin{cases}\frac{a_{1} \log a_{1}-a_{2} \log a_{2}}{a_{1}-a_{2}}-\log e^{\gamma} & \text { if } a_{1} \neq a_{2} \\
\log a_{1}+\log e^{1-\gamma} & \text { if } a_{1}=a_{2},\end{cases}
\end{aligned}
$$

where $a_{1}$ and $a_{2}$ are positive scalar constants and $\gamma$ is Euler's constant.

Applying formula (28), the first term and the second term inside the $\min \{$.$\} in expression (26) are given,$ respectively, by

$$
E\left[\log _{2}\left(\beta P\left(\gamma_{1}+\gamma_{2}\right)\right)\right]=\log _{2} P+\log _{2} \beta+\log _{2} e^{1-\gamma},
$$

and

$$
\begin{aligned}
E\left[\log _{2}\left(\beta P \gamma_{1}+(1-\beta) P g \gamma_{3}\right)\right]= & \log _{2} P \\
& +\frac{g(1-\beta) \log _{2}(g(1-\beta))-\beta \log _{2} \beta}{g(1-\beta)-\beta}-\log _{2} e^{\gamma} .
\end{aligned}
$$

Expression (29) is an increasing function of $\beta$. It is easy to show that expression (30) is a decreasing function of $\beta$ (for $g$ of interest, i.e., $g>1$ ). Thus the optimal value $\beta^{*}$ solution of the maximization problem (27) can be found by equating expressions (29) and (30) as

$$
\begin{aligned}
\log _{2} P+\log _{2} \beta^{*}+\log _{2} e^{1-\gamma}= & \log _{2} P \\
& +\frac{g\left(1-\beta^{*}\right) \log _{2}\left(g\left(1-\beta^{*}\right)\right)-\beta^{*} \log _{2} \beta^{*}}{g\left(1-\beta^{*}\right)-\beta^{*}}-\log _{2} e^{\gamma}
\end{aligned}
$$

Equation 31 is equivalent to

$$
\ln \left(\beta^{*}\right)+1=\frac{g\left(1-\beta^{*}\right) \ln \left(g\left(1-\beta^{*}\right)\right)-\beta^{*} \ln \left(\beta^{*}\right)}{g\left(1-\beta^{*}\right)-\beta^{*}},
$$

where $\ln (x)$ is the natural logarithm of $x$.

After some algebraic manipulations (32) can be rewritten equivalently as

$$
\frac{-\beta^{*}}{g\left(1-\beta^{*}\right)} \exp \left(\frac{-\beta^{*}}{g\left(1-\beta^{*}\right)}\right)=-\frac{1}{e} \text {. }
$$

It can be recognized that Equation 33 is in the form of a transcendental Equation 1. Thus we have

$$
W\left(-\frac{1}{e}\right)=\frac{-\beta^{*}}{g\left(1-\beta^{*}\right)} .
$$

The optimal value $\beta^{*}$ is deduced from (34) as

$$
\beta^{*}=\frac{K g}{K g+1},
$$


where $K=-W\left(-\frac{1}{e}\right)=1$.

It is interesting is to observe that the optimal power allocation is obtained in closed-form and depends only on $g$, i.e., on the distance $d$ between the relay node and the destination node and the path-loss power attenuation exponent $\alpha$.

\subsection{Comparison with CSIT-based power allocation}

In order to assess the relevance of the CDIT-based approach, it has to be compared to the allocation scheme based on perfect CSIT. Perfect CSIT is unrealistic, but for the purpose of comparison, let us assume perfect CSIT. Then the power allocation can be formulated to maximize the instantaneous capacity instead of the ergodic capacity. Mathematically, the CSIT-based power allocation can be formulated as

$$
\begin{aligned}
\max _{0 \leq \rho, \beta \leq 1} \min \{ & \frac{1}{2} \log _{2}\left(1+\beta P\left(1-\rho^{2}\right)\left(\gamma_{1}+\gamma_{2}\right)\right), \\
& \left.\frac{1}{2} \log _{2}\left(1+\beta P \gamma_{1}+((1-\beta) g+2 \rho \sqrt{\beta(1-\beta) g}) P \gamma_{3}\right)\right\} .
\end{aligned}
$$

The optimal values of $\rho$ and $\beta$ solution of (36) have been found in [11] as

$$
\begin{aligned}
& \rho^{*}=\frac{1}{\sqrt{g^{2}+2 g+2}}, \\
& \beta^{*}=\frac{g^{2}+2 g+2}{g^{2}+3 g+2} .
\end{aligned}
$$

The instantaneous capacity upper bound for high SNR regime is deduced as

$$
C_{\text {inst }}=\log _{2}\left(\frac{2(g+1)}{g+2} P\right) .
$$

Both CDIT-based and CSIT-based optimal power allocation expressions (35) and (38) are in closed-form and very fast to compute. Thus, the complexity is almost the same. The main difference between the two allocation schemes is the amount of feedback required to perform power allocation. Recall that, for the CSIT-based scheme, the allocation is performed after each symbol period. Let $N_{\mathrm{s}}$ be the number of symbol periods after which the CDIT-based resource allocation is performed. Then a rough estimation tells us that the feedback needed to perform CDIT-based power allocation is reduced by $\frac{1}{N_{\mathrm{s}}}$ compared to the perfect CSIT scheme.

\section{Simulation results}

In order to assess the performance of the proposed resource allocation methods, we conduct simulations and compare the simulation results with other baseline schemes.

\subsection{Simulation results for wireless mesh networks}

We consider a cluster with four wireless nodes with the scheduling tree topology shown in Figure 4 and 4 a total number of subcarriers $N=128$ over a $1-\mathrm{MHz}$ band. The relative effective SNR difference between MC 1 (the closest MC to the MR) and MC 2, 3, and 4 are 3, 6, and $10 \mathrm{~dB}$, respectively. The minimum rate requirements are chosen to be the same for all MCs, the maximal power at each MC $k$ is $\bar{p}_{k}=50 \mathrm{~mW}$, the thermal noise power is $\sigma_{n}^{2}=10^{-11} \mathrm{~W}$.

We assume a Rayleigh fading. Thus, for the CDI-based allocation, the $\alpha_{k}$ follow a $\chi^{2}$ distribution with $2 L_{k}$ degree of freedom, where $L_{k}$ is the number of outgoing links at MC $k$. For MC with a single outgoing link, $\alpha_{k}$ is reduced to an exponential distribution.

We name the proposed scheme with optimal allocation at MR and MCs as full optimal resource allocation (FORA). For comparison, we also implement the following resource allocation schemes:

1. MR-based optimal resource allocation (MORA) where the MR performs the proposed optimal subcarrier assignment, but each MC performs uniform power allocation among its outgoing links.

2. Full uniform resource allocation (FURA) where each $M C$ is assigned the same number of subcarriers and transmit power at each $\mathrm{MC}$ is uniformly distributed over the assigned subcarriers and the active links.

We evaluate system performance in terms of sum rate, and satisfaction of minimum rate requirements.

In Figure 5, the performance of the proposed FORA is compared to that of the optimal resource allocation at MR under uniform power allocation at MCs (MORA) and the FURA. The result shows that the proposed optimal resource allocation brings significant gain over uniform resource allocation, especially for low SNRs.

Figure 6 shows the user's rate for different allocation schemes when the users minimum data rate demands are constrained to $R_{k}=1 \mathrm{Mbps}$ for all MCs. We observe that under optimal allocation, the need of all users in terms of data rate is satisfied. This is not the case under uniform allocation. With uniform allocation, there is an over-allocation for closer MCs to the MR (MCs 1 and 2) while the rate demand of farer users with bad channel conditions (MCs 3 and 4) are not satisfied. 


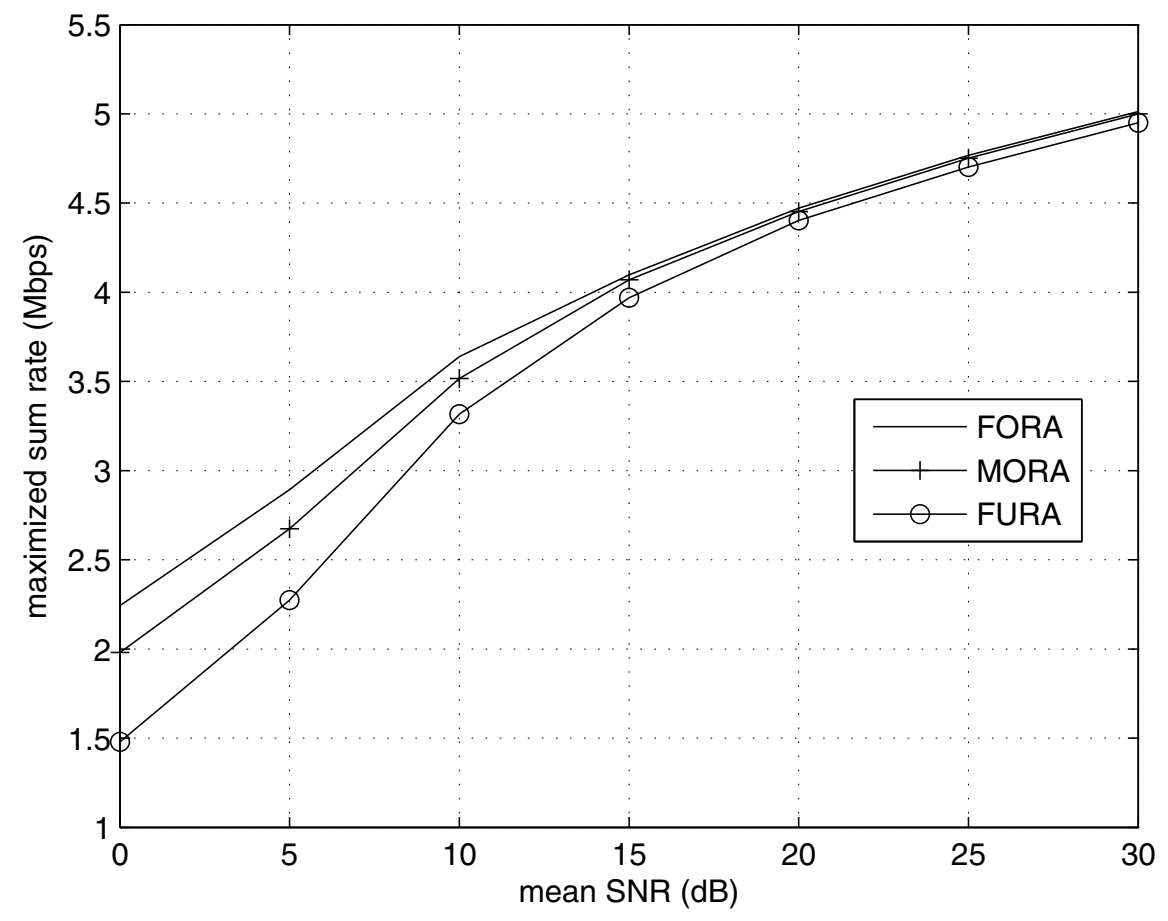

Figure 4 Network topology used in the simulations.

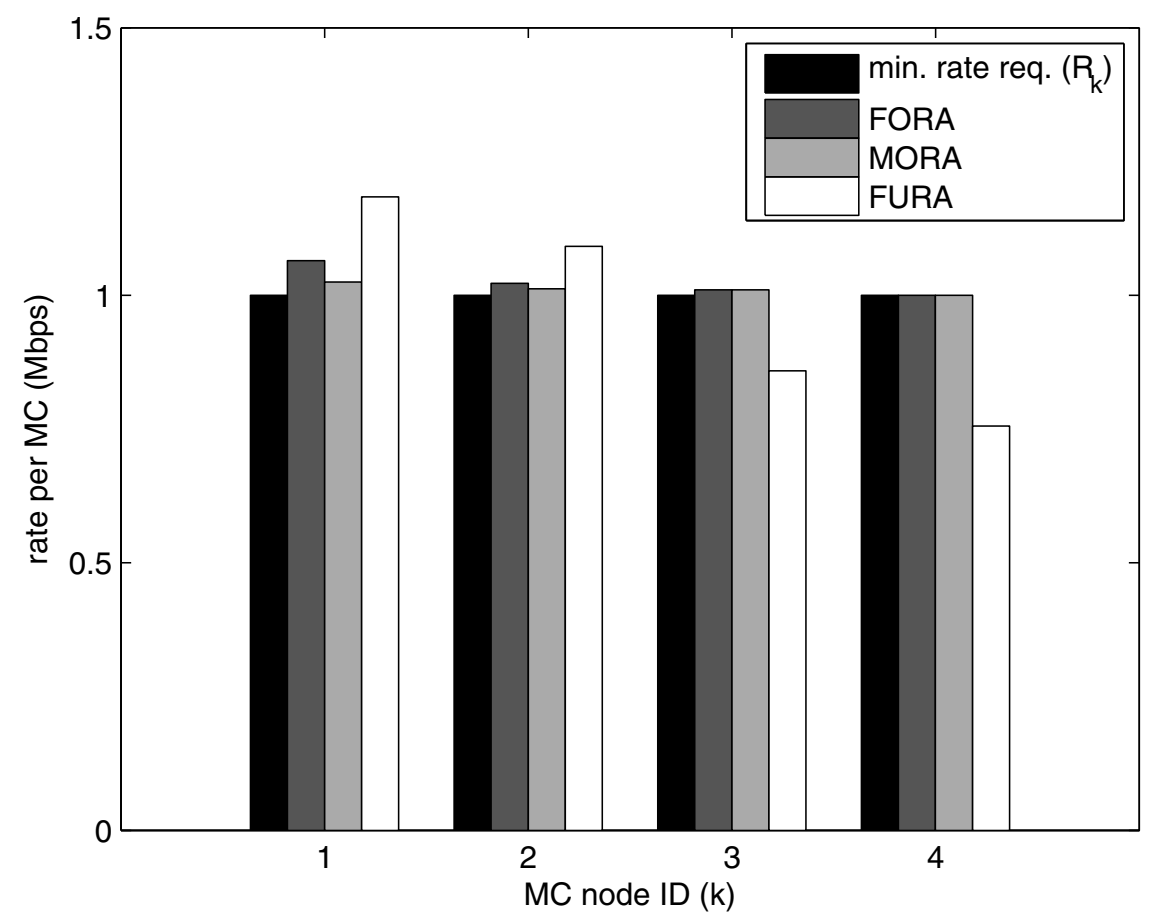

Figure 5 Maximized sum rate versus mean SNR for various resource allocation schemes. 


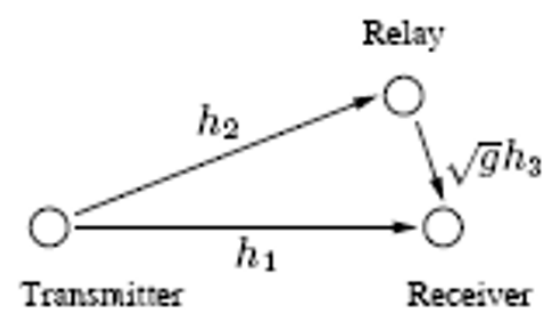

Figure 6 Per mesh client rate for $R_{k}=1 \mathrm{Mbps}$

\subsection{Simulation results for relay networks}

In all the simulations, we assume a path-loss power attenuation exponent of 2 , and hence $g=1 / d^{2}$. The distance $d$ between the relay node and the receiver node varies from 0.1 to 1 .

In Figure 7, the ergodic capacity achieved using the proposed power allocation scheme is compared to the one obtained with uniform power allocation $(\beta=0.5$, $\forall d \in[0.1,1])$. We consider system average network power constraints of $P=10$ and $P=100$. It can be observed that the achieved capacity using the proposed optimal power allocation method outperforms the capacity obtained with uniform allocation.

Figure 8 illustrates the achieved capacity using the proposed CDIT-based optimal power allocation (35) in comparison with the capacity of the CSIT-based optimal power allocation (38). The CSIT-based capacity is averaged over the same number of channel realizations $N_{\mathrm{s}}$ over which the distribution is taken to evaluate the ergodic capacity. The result shows that the gap between the average capacity and the ergodic capacity is small. Thus, even with CDIT only, optimal power allocation improves performance of relay networks.

The trade-off between reduced feedback and performance degradation of the proposed CDIT-based optimal power allocation in comparison with the perfect CSITbased optimal power allocation is shown in Figure 9. We observe that adapting the transmission strategy to the short-term channel statistics, increases the performance but also increases the amount of feedback. However, if the transmission is adapted to the long-term channel statistics, the amount of feedback decreases significantly but with a penalty on the performance. For a CDIT-based allocation with a distribution taken over 16

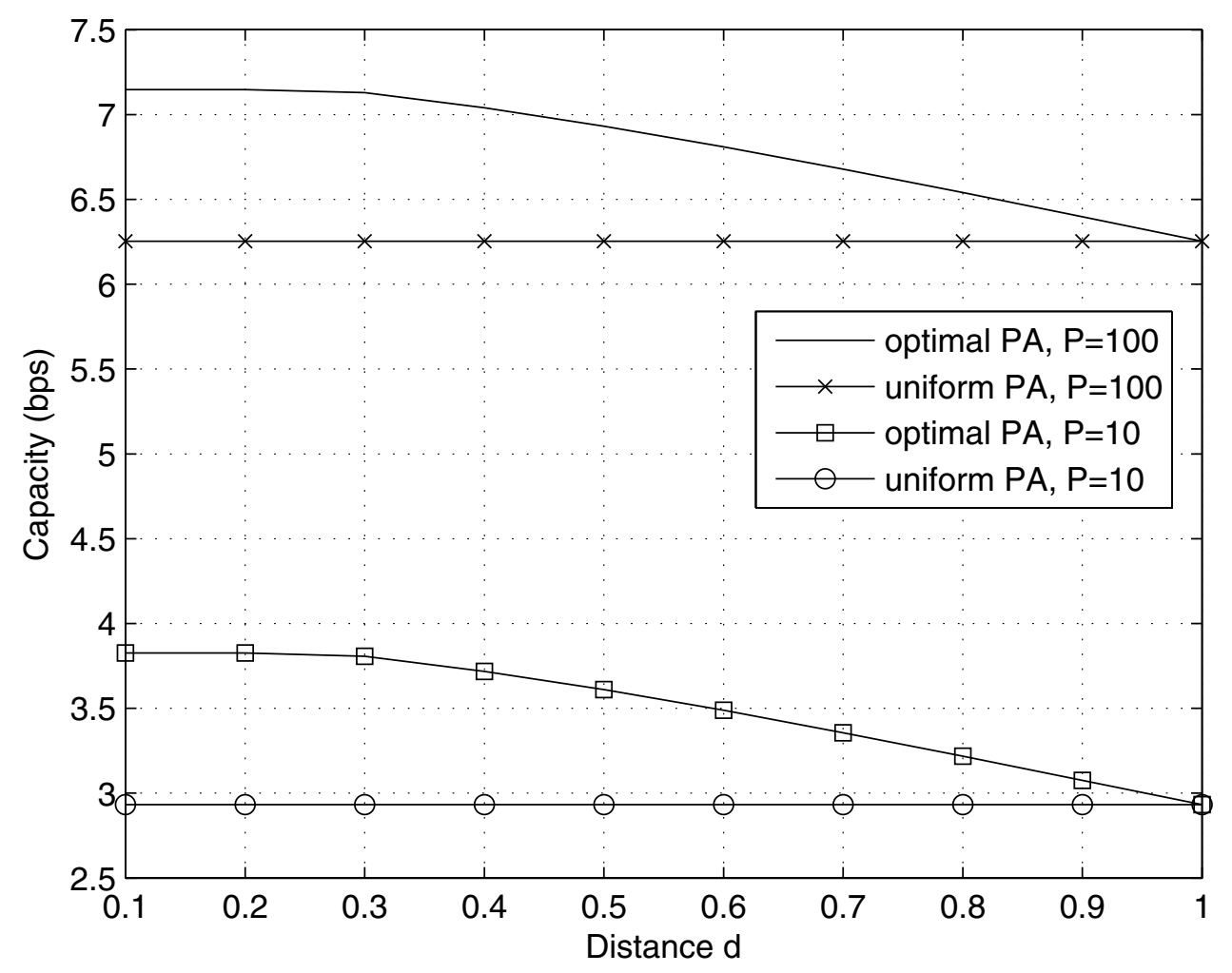

Figure 7 Maximized capacity versus distance $d$ for CDIT-based optimal power allocation and uniform power allocation. 


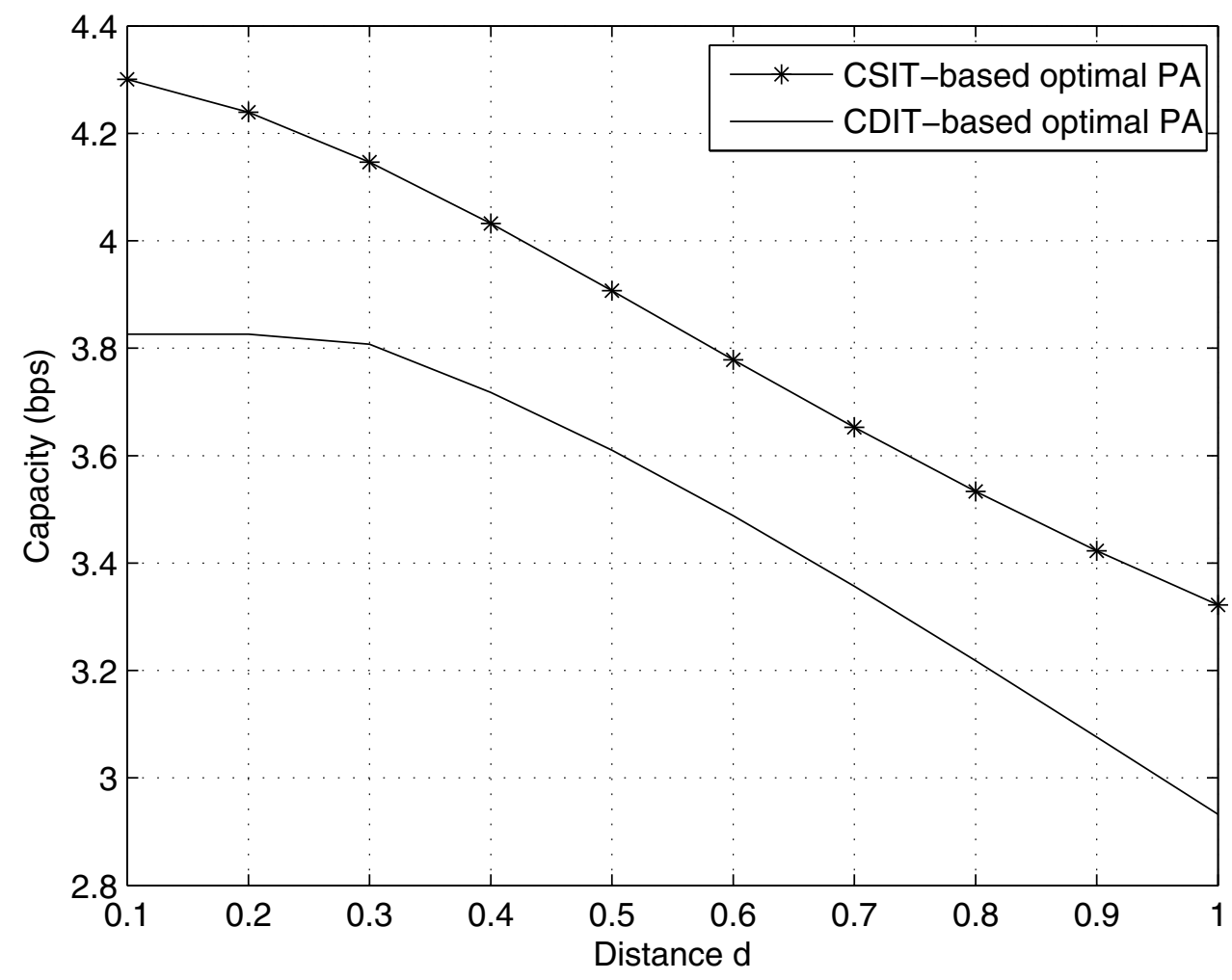

Figure 8 Maximized capacity versus distance $d$ for CDIT-based optimal power allocation and CSIT-based optimal power allocation.

symbol periods, the amount of feedback is reduced by 93.75\%, while the performance degradation in terms of capacity is less than $12 \%$.

\section{Conclusion}

We have addressed constrained resource allocation problems for wireless mesh and relay networks. For mesh networks, we have formulated a distributed subcarrier allocation problem to maximize the sum rate while satisfying minimum rate demand. For relay networks, we have formulated power allocation problem to maximize the ergodic capacity under total power constraint. Both cases of perfect and statistical channel knowledge at the transmitter have been considered. We have shown how the Lambert-W function can be use to efficiently find the optimal resource allocation in closedform. Using the Lambert-W function, resource allocation can quickly be determined since a number of popular mathematical softwares, including Maple and Matlab, contain the Lambert-W function as an optimization component. The Lambert-W function can be combined with the Lagrange dual approach to solve variety of wired and wireless resource allocation problems without resorting to complex numerical algorithms.

\section{Appendix}

Appendix A: Derivation of (8)

Equation 7 can be rewritten as

$$
\ln \left(\frac{n_{k}}{n_{k}+\alpha_{k}}\right)-\frac{n_{k}}{n_{k}+\alpha_{k}}+1+\frac{\mu}{\frac{B}{\ln 2}\left(1+\lambda_{k}\right)}=0 .
$$

Equation A.1 can be rewritten equivalently as

$$
-\frac{n_{k}}{n_{k}+\alpha_{k}} e^{\left(-\frac{n_{k}}{n_{k}+\alpha_{k}}\right)}=-\exp \left(-1-\frac{\mu}{\frac{B}{\ln 2}\left(1+\lambda_{k}\right)}\right)
$$

Expression (A.2) is in the form of the Lambert-W function $W(x)$, which is the solution to $W(x) e^{W(x)}=x$. Thus, from (A.2) we can deduce that

$$
W\left(-\exp \left(-1-\frac{\mu}{\frac{B}{\ln 2}\left(1+\lambda_{k}\right)}\right)\right)=-\frac{n_{k}}{n_{k}+\alpha_{k}}
$$

which when then solved for $n_{k}$ gives (8). 


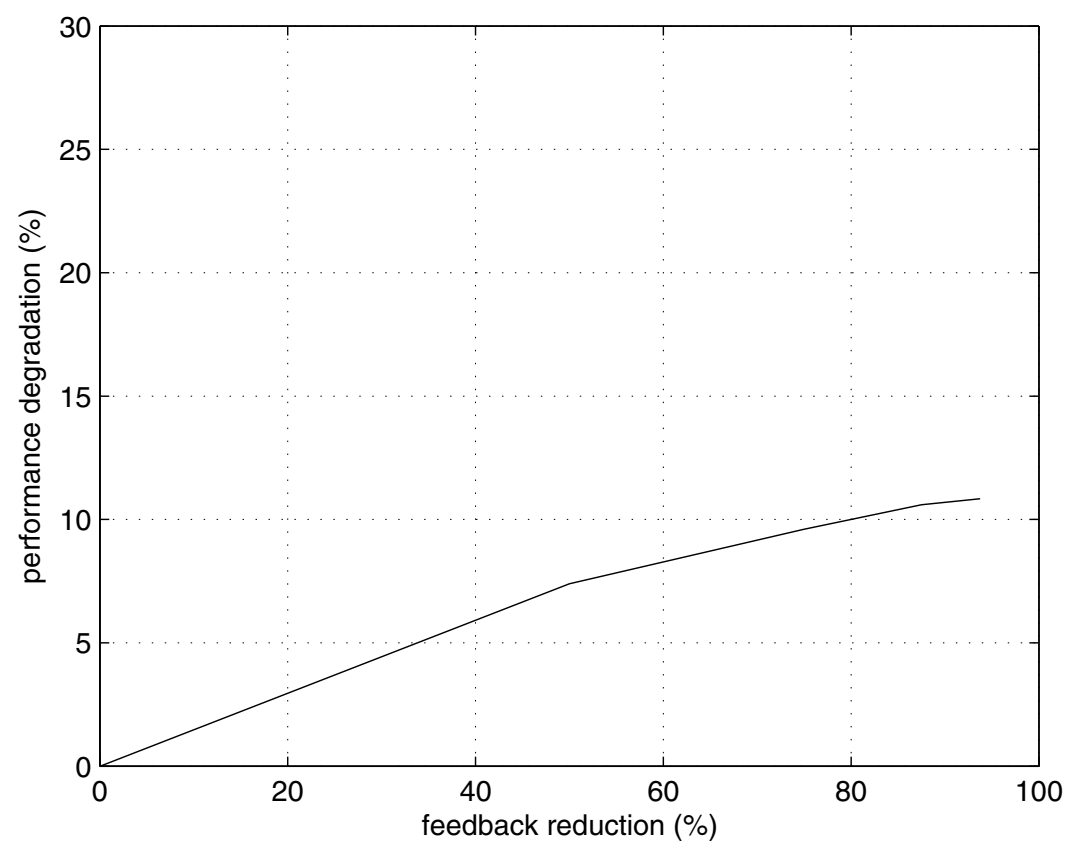

Figure 9 Trade-off between feedback reduction and performance degradation of the CDI-based allocation compared with the perfect CSIT-based allocation

\section{Appendix B: Derivation of (10)}

Let $w_{k}\left(\lambda_{k}, \mu\right)=W\left(-\exp \left(-1-\frac{\mu}{\frac{B}{\ln 2}\left(1+\lambda_{k}\right)}\right)\right)$.

Replacing $n_{k}$ in (6) by its optimal value given by (8), we get the dual function

$$
\begin{aligned}
L\left(\lambda_{k}, \mu\right)= & \sum_{k=1}^{K} \frac{-\alpha_{k} w_{k}\left(\lambda_{k}, \mu\right)}{1+w_{k}\left(\lambda_{k}, \mu\right)} B \log _{2}\left(-\frac{1}{w_{k}\left(\lambda_{k}, \mu\right)}\right) \\
& +\sum_{k=1}^{K} \lambda_{k}\left(\frac{-\alpha_{k} w_{k}\left(\lambda_{k}, \mu\right)}{1+w_{k}\left(\lambda_{k}, \mu\right)} B \log _{2}\left(-\frac{1}{w_{k}\left(\lambda_{k}, \mu\right)}\right)-R_{k}\right)( \\
& -\mu\left(\sum_{k=1}^{K} \frac{-\alpha_{k} w_{k}\left(\lambda_{k}, \mu\right)}{1+w_{k}\left(\lambda_{k}, \mu\right)}-N\right)
\end{aligned}
$$

Equation B.1 can be rewritten as

$$
\begin{aligned}
L\left(\lambda_{k}, \mu\right)= & \mu N-\sum_{k=1}^{K} \lambda_{k} R_{k}-\mu \sum_{k=1}^{K} \frac{-\alpha_{k} w_{k}\left(\lambda_{k}, \mu\right)}{1+w_{k}\left(\lambda_{k}, \mu\right)} \\
& +\sum_{k=1}^{K}\left(\left(\lambda_{k}+1\right) \frac{\alpha_{k} w_{k}\left(\lambda_{k}, \mu\right)}{1+w_{k}\left(\lambda_{k}, \mu\right)} B \log _{2}\left(-w_{k}\left(\lambda_{k}, \mu\right)\right)\right)
\end{aligned}
$$

Applying (2) for $n=1$ and using the property of the derivative of a composite function, we obtain the derivative of $w_{k}$ with respect to $\lambda_{k}$ for fixed $\mu$ as

$$
\frac{\mathrm{d} w_{k}\left(\lambda_{k}, \mu\right)}{\mathrm{d} \lambda_{k}}=\frac{w_{k}\left(\lambda_{k}, \mu\right)}{1+w_{k}\left(\lambda_{k}, \mu\right)} \cdot \frac{\mu \ln 2}{B} \cdot \frac{1}{\left(1+\lambda_{k}\right)^{2}} .
$$

Using (B.3), we calculate the derivative of $L\left(\lambda_{k}, \mu\right)$ with respect to $\lambda_{k}$ for fixed $\mu$ as

$$
\begin{aligned}
\frac{\mathrm{d} L\left(\lambda_{k}, \mu\right)}{\mathrm{d} \lambda_{k}}= & -R_{k}+\frac{B}{\ln 2} \cdot \frac{\alpha_{k} w_{k}\left(\lambda_{k}, \mu\right)}{1+w_{k}\left(\lambda_{k}, \mu\right)} \cdot \ln \left(-w_{k}\left(\lambda_{k}, \mu\right)\right) \\
& +\frac{\mu}{1+\lambda_{k}} \cdot \frac{\alpha_{k} w_{k}\left(\lambda_{k}, \mu\right)}{1+w_{k}\left(\lambda_{k}, \mu\right)} \cdot \ln \left(-w_{k}\left(\lambda_{k}, \mu\right)\right) \\
& +\frac{\mu}{1+\lambda_{k}} \cdot \frac{\alpha_{k} w_{k}\left(\lambda_{k}, \mu\right)}{\left(1+w_{k}\left(\lambda_{k}, \mu\right)\right)^{2}} \\
& +\frac{\mu^{2} \ln 2}{B\left(1+\lambda_{k}\right)^{2}} \cdot \frac{\alpha_{k} w_{k}\left(\lambda_{k}, \mu\right)}{\left(1+w_{k}\left(\lambda_{k}, \mu\right)\right)^{3}} .
\end{aligned}
$$

Applying KKT optimality conditions, we set the derivative (B.4) to zero to obtain

$$
\begin{aligned}
-\frac{R_{k}}{\alpha_{k}} & +\frac{B}{\ln 2} \cdot \frac{w_{k}\left(\lambda_{k^{\prime}}^{*} \mu\right)}{1+w_{k}\left(\lambda_{k^{\prime}}^{*} \mu\right)} \cdot \ln \left(-w_{k}\left(\lambda_{k^{\prime}}^{*} \mu\right)\right) \\
& +\frac{\mu}{1+\lambda_{k}^{*}} \cdot \frac{w_{k}\left(\lambda_{k^{\prime}}^{*} \mu\right)}{1+w_{k}\left(\lambda_{k^{\prime}}^{*} \mu\right)} \cdot \ln \left(-w_{k}\left(\lambda_{k^{\prime}}^{*} \mu\right)\right) \\
& +\frac{\mu}{1+\lambda_{k}^{*}} \cdot \frac{w_{k}\left(\lambda_{k^{\prime}}^{*} \mu\right)}{\left(1+w_{k}\left(\lambda_{k^{\prime}}^{*} \mu\right)\right)^{2}} \\
& +\frac{\mu^{2} \ln 2}{B\left(1+\lambda_{k}^{*}\right)^{2}} \cdot \frac{w_{k}\left(\lambda_{k^{\prime}}^{*} \mu\right)}{\left(1+w_{k}\left(\lambda_{k^{\prime}}^{*} \mu\right)\right)^{3}}=0,
\end{aligned}
$$

where $\lambda_{k}^{*}$ is the optimal value of $\lambda_{k}$. 
We see that Equation B.5 can be rewritten in the form of Equation 10.

\section{Appendix C: Proof of Proposition 1}

Let

$$
g_{k, m}(\mu)=\frac{-\alpha_{k} \tilde{w}_{k}(\mu)}{1+\tilde{w}_{k}(\mu)}
$$

where

$$
\tilde{w}_{k}(\mu)=W\left(-\exp \left(-1-\frac{\mu}{\frac{B}{\ln 2}\left(1+\lambda_{k}^{*}\right)}\right)\right) .
$$

The derivative of $\tilde{w}_{k}$ with respect to $\mu$ is given by

$$
\frac{\mathrm{d} w_{k}(\mu)}{\mathrm{d} \mu}=\frac{w_{k}(\mu)}{1+w_{k}(\mu)} \cdot\left(-\frac{1}{\frac{B}{\ln 2}\left(1+\lambda_{k}^{*}\right)}\right) .
$$

Using (C.1), we can calculate the derivative of $g_{k, m}(\mu)$ with respect to $\mu$ as

$$
\frac{\mathrm{d} g_{k, m}(\mu)}{\mathrm{d} \mu}=\frac{\alpha_{k} \cdot \frac{w_{k}(\mu)}{1+w_{k}(\mu)} \cdot\left(-\frac{1}{\frac{B}{\ln 2}\left(1+\lambda_{k}^{*}\right)}\right)}{\left(1+w_{k}(\mu)\right)^{2}}<0 .
$$

Thus

$$
\frac{\mathrm{d} g_{m}(\mu)}{\mathrm{d} \mu}=\sum_{k=1}^{K} \frac{\mathrm{d} g_{k, m}(\mu)}{\mathrm{d} \mu}<0 .
$$

namely, $g_{m}(\mu)$ is a strictly decreasing function of $\mu$. This completes the proof.

\section{Abbreviations}

BS: base station; BER: bit-error-rate; CDI: channel distribution information; CSI: channel state information; cdf: cumulative distribution function; FORA: ful optimal resource allocation; FURA: full uniform resource allocation; MC: mesh clients; MR: mesh router; MS: mobile station; MORA: MR-based optimal resource allocation; RS: relay station; WMNs: wireless Mesh networks; ZMCSCG: zero mean circularly symmetric complex Gaussian.

\section{Author details}

'ICTEAM Institute, Université Catholique de Louvain, Place du Levant 2, 1348 Louvain-la-Neuve, Belgium ${ }^{2}$ SYSCOM, Université Paris-Est, Marne la Vallée, France

\section{Competing interests}

The authors declare that they have no competing interests.

Received: 4 October 2010 Accepted: 20 June 2011

Published: 20 June 2011

\section{References}

1. A Nosratinia, A Hedayat, Cooperative communication in wireless networks. IEEE Commun Mag. 42(10):74-80 (2004). doi:10.1109/MCOM.2004.1341264

2. JN Laneman, D Tse, GW Wornell, Cooperative diversity in wireless networks: efficient protocols and outage behavior. IEEE Trans Inf Theory. 51(9):3062-3080 (2004)
3. G Kramer, M Gastpar, P Gupta, Cooperative strategies and capacity theorems for relay networks. IEEE Trans Inf Theory. 51(9):3037-3063 (2005) doi:10.1109/TIT.2005.853304

4. IF Akyildiz, X Wang, W Wang, Wireless mesh networks: a survey. Comput Netw ISDN Syst. 47(4):445-487 (2005)

5. KD Lee, VCM Leung, Fair allocation of subcarrier and power in an OFDMA wireless mesh networks. IEEE J Sel Areas Commun. 24(11):2051-2060 (2006)

6. HT Cheng, W Zhuang, Novel packet-level resource allocation with effective QoS provision for Wireless Mesh Networks. IEEE Trans Wireless Commun. 8(2):694-700 (2009)

7. A Host-Madsen, J Zhang, Capacity bounds and power allocation in wireless relay channel. IEEE Trans Inf Theory. 51(6):2020-2040 (2005). doi:10.1109/ TIT.2005.847703

8. Y Yao, X Cai, GB Giannakis, On energy efficient and optimum resource allocation of relay transmissions. IEEE Trans Wireless Commun. 4(6):2917-2927 (2005)

9. F Brah, J Louveaux, L Vandendorpe, CDIT-Based constrained resource allocation for mobile WiMAX systems. EURASIP J Wireless Commun Netw 2009, 1-8 (2009). Article ID 425367

10. D Grunduz, E Erkip, Opportunistic cooperation by dynamic resource allocation. IEEE Trans Wireless Commun. 6(4):1446-1454 (2007)

11. CTK Ng, AJ Goldsmith, The impact of CSI and power allocation on relay channel capacity and cooperation strategies. IEEE Trans Wireless Commun. 7(12):5380-5389 (2008)

12. RM Corless., et al, On the Lambert W function. Adv Comput Math. 5 , 329-359 (1996). doi:10.1007/BF02124750

13. N Galidakis, On an application of Lambert's W function to infinite exponentials complex variables. Elliptic Equ. 49(11):759-780 (2004)

14. P Hruska, Z Chobola, L Grmela, Diode I-U curve fitting with Lambert W function. Proceedings of the IEEE International Conference on Microelectronics, Belgrade. 468-471 (2006)

15. P Sengupta, The Lambert-W function and solutions to Kepler's equation Clestial Mech Dyn Astron. 99(1):13-22 (2007). doi:10.1007/s10569-007-9085-6

16. RL Graham, DE Knuth, O Patashnik, Concrete Mathematics: A Foundation for Computer Sciences,, 2nd edn. (Addison-Wesley, Reading, 1994)

17. S Boyd, L Vandenberghe, Convex Optimization. (Cambridge University Press, Cambridge, 2004)

doi:10.1186/1687-1499-2011-19

Cite this article as: Brah et al:: On the Lambert-W function for constrained resource allocation in cooperative networks. EURASIP Journal on Wireless Communications and Networking 2011 2011:19.

\section{Submit your manuscript to a SpringerOpen ${ }^{\circ}$ journal and benefit from:}

- Convenient online submission

- Rigorous peer review

- Immediate publication on acceptance

- Open access: articles freely available online

- High visibility within the field

- Retaining the copyright to your article

Submit your next manuscript at $>$ springeropen.com 